\title{
Development of antibiotics that dysregulate the Neisserial ClpP protease
}

Gursonika Binepal $^{1, \ddagger}$, Mark F. Mabanglo ${ }^{1, \ddagger}$, Jordan D. Goodreid ${ }^{2, \ddagger}$, Elisa Leung ${ }^{1, \ddagger}$, Marim M. Barghash $^{1}$, Keith S. Wong ${ }^{1}$, Funing Lin ${ }^{2}$, Michele Cossette ${ }^{2}$, Jazmin Bansagi ${ }^{2}$, Boxi Song ${ }^{2}$, Vitor Hugo Balasco Serrão ${ }^{3}$, Emil F. Pai ${ }^{1,4,5, * *}$, Robert A. Batey ${ }^{2, * *}$, Scott D. Gray-Owen ${ }^{6, * *}$, and Walid A. Houry $1,2, * *, \dagger$

${ }^{1}$ Department of Biochemistry, University of Toronto, Toronto, Ontario M5G 1M1, Canada.

${ }^{2}$ Department of Chemistry, University of Toronto, Toronto, Ontario M5S 3H6, Canada.

${ }^{3}$ Department of Laboratory Medicine and Pathobiology, University of Toronto, Toronto, ON M5S 1A8, Canada.

${ }^{4}$ Department of Medical Biophysics, University of Toronto, Toronto, Ontario M5S 1A8, Canada. ${ }^{5}$ Ontario Cancer Institute/Princess Margaret Hospital, Toronto, Ontario M5G 1L7, Canada.

${ }^{6}$ Department of Molecular Genetics, University of Toronto, Toronto, Ontario M5S 1A8, Canada.

These authors contributed equally to this work.

*** Equally contributing senior authors.

${ }^{\dagger}$ Corresponding author. W. A. Houry, 661 University Avenue, MaRS Centre, West Tower, Room 1612, Toronto, ON M5G 1M1, Canada. Tel.: (416) 946-7141; Fax: (416) 978-8548; Email: walid.houry@utoronto.ca 
A

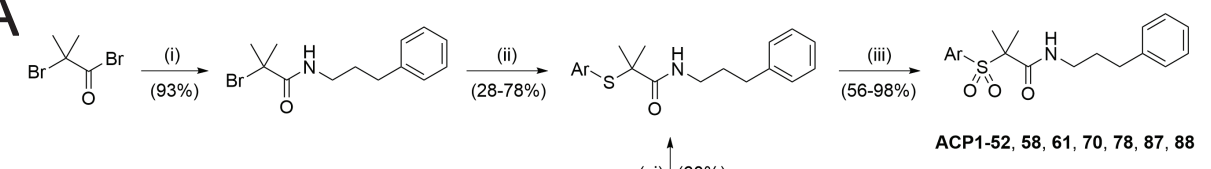

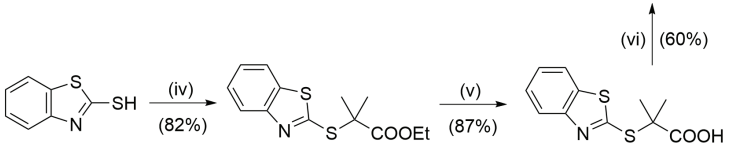

(i)

$\begin{array}{lllll}X=\mathrm{NO}_{2}, \mathrm{Y}=\mathrm{H} & \mathrm{ACP1} 1-21 & \mathrm{X}=\mathrm{NH}_{2}, \mathrm{Y}=\mathrm{H} & \mathrm{ACP1} 111 & \mathrm{ACP1}-27,55,63,65,69,72,82,85,90 \\ \mathrm{X}=\mathrm{H}_{2} \mathrm{Y}=\mathrm{NO}_{2} & { }_{\mathrm{ACP} 1-37} & \mathrm{X}=\mathrm{H}, \mathrm{Y}=\mathrm{NH}_{2} & \text { ACP1-08 } & \end{array}$

Reagents and conditions: (i) $\mathrm{H}_{2} \mathrm{~N}\left(\mathrm{CH}_{2}\right)_{3} \mathrm{Ph}$ (1.0 equiv), $-\mathrm{PrNEt} \mathrm{Pr}_{2}\left(1.2\right.$ equiv), THF, $0^{\circ} \mathrm{C}$ to rt, $24 \mathrm{~h}$; (ii) Ar-SH (1.0 equiv), KOH (1.0-1.2 equiv) or $\mathrm{K}_{2} \mathrm{CO}_{3}$ (1.3-1.6 equiv), EtOH, reflux, 17-67 $\mathrm{h}$; (iii) $m \mathrm{mCPBA}\left(2.2-2.7\right.$ equiv), $\mathrm{NaHCO}_{3}\left(3.5-12.7\right.$ equiv), $\mathrm{CH}_{2} \mathrm{Cl}_{2}, 0{ }^{\circ} \mathrm{C}$ to $\mathrm{rt}, 24-29 \mathrm{~h}$; (iv) $\mathrm{H}_{2} \mathrm{~N}\left(\mathrm{CH}_{2}\right)_{3} \mathrm{Ph}$ (1.0 equiv), PyBOP (1.1 equiv), $i-\mathrm{PrNEt}_{2}\left(2.2\right.$ equiv), DMF, $0^{\circ} \mathrm{C}$ to $\mathrm{rt}, 24 \mathrm{~h}$.

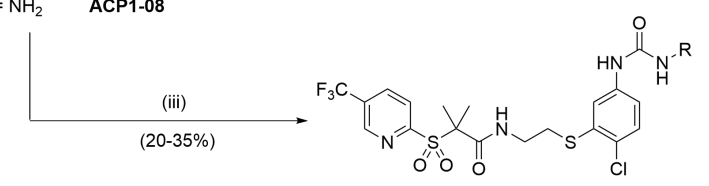

$\begin{array}{ll}R=H & A C P 1-80 \\ R=P h & A C P 1-81\end{array}$

Reagents and conditions: (i) $\mathrm{SnCl}_{2} \cdot 2 \mathrm{H}_{2} \mathrm{O}$ (10.5 equiv), EtOAc, reflux, $4-19 \mathrm{~h}$; (ii) $\mathrm{CDI}$ (1.05 eq), $\mathrm{CH}_{2} \mathrm{Cl}_{2}, \mathrm{rt}, 20 \mathrm{~h}$ then amine (1.0 equiv) or PhSH (1.0 equiv) or PhOH (1.0 equiv), ${ }^{\circ} \mathrm{C}$ to $\mathrm{rt}, 5 \mathrm{~min}$; (iii) Phenyl isocyanate (1.2 equiv) or trimethylsilyl isocyanate (1.4 equiv), $\mathrm{CH}_{2} \mathrm{Cl}_{2}, \mathrm{rt}, 5 \mathrm{~h}$.

$B_{0}$

$$
\text { (iv) }
$$

Reagents and conditions: (i) $\mathrm{NaH}\left(1.1-1.2\right.$ equiv), $\mathrm{THF}, 0{ }^{\circ} \mathrm{C}, 20$ min then $\mathrm{BrCH}_{2} \mathrm{COOBn}$ (1.0 equiv), $0{ }^{\circ} \mathrm{C}$ to $\mathrm{rt}, 2-17 \mathrm{~h}$; (ii) $m \mathrm{CPBA}$ (2.2-2.4 equiv), $\mathrm{NaHCO}_{3}$ (2.1-7.6 eq), $\mathrm{CH}_{2} \mathrm{Cl}_{2}, 0^{\circ} \mathrm{C}$ to rt, $24 \mathrm{~h}$; (iii) BrCH $\left.\mathrm{Br}_{2} \mathrm{CH}_{2}\right)_{1} \mathrm{Br}$ (11.1-1.2 equiv, where $\left.n=0-3\right), \mathrm{K}_{2} \mathrm{CO}_{3}$ (5.9-8.8 equiv), MeCN, reflux, $6-24 \mathrm{~h}$; (iv) Pd/C "Note: MeOH:THF (3:1) was used as the solvent system for $X=N$ and $n=3$. THF was used as the solvent for $X=C H$ and $n=2$.

C

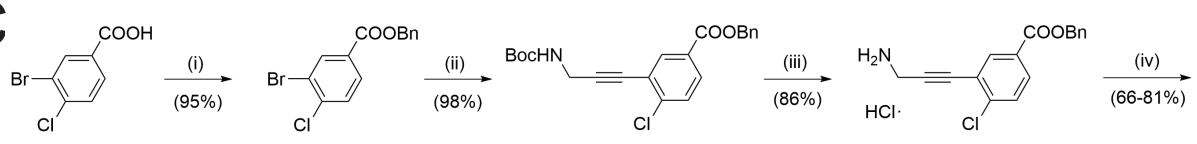

$$
\text { (v) }
$$

Reagents and conditions: (i) $\mathrm{NBu}_{4}$ ( $\left(10 \mathrm{~mol} \%\right.$ ), $\mathrm{BnBr}$ (1.1 equiv), $\mathrm{NEt}_{3}(1.2$ equiv), $\mathrm{THF}, \mathrm{tt}, 12 \mathrm{~h}$; (ii) $\mathrm{N}$-Boc-propargylamine (1.0 equiv), $\left.\mathrm{Pd} \mathrm{PPh}_{3}\right)_{4}(10 \mathrm{~mol} \%), \mathrm{Cul}(5 \mathrm{~mol} \%), \mathrm{NEt}_{3}(2.1 \mathrm{equiv}), \mathrm{THF}, 60{ }^{\circ} \mathrm{C}, 38 \mathrm{~h}$, sealed tube; (iii) $\mathrm{HCl}(10.0$ equiv), dioxane, rt, $24 \mathrm{~h}$; (iv) $\mathrm{ArSO}_{2} \mathrm{C}\left(\mathrm{CH}_{3}\right)_{2} \mathrm{COOH}$ (1.0 equiv), PyBOP (1.1 equiv), i- $-\mathrm{Pr}_{2} \mathrm{NEt}\left(2.1\right.$ equiv), DMF, $0^{\circ} \mathrm{C}$ to o th, $15 \mathrm{~h}$; (v) Pd/C (10-12 mol \%), $\mathrm{H}_{2}(1 \mathrm{~atm}$ ), THF, $\mathrm{rt}, 15-17 \mathrm{~h}$; (vi) $\mathrm{R}_{1} \mathrm{R}_{2} \mathrm{NH}$ (1.1-2.1 equiv), PyBOP (1.1 equiv), $i-\mathrm{Pr}_{2} \mathrm{NEt}\left(2.5-2.7\right.$ equiv), DMF, $0{ }^{\circ} \mathrm{C}$ to $\mathrm{rt}, 15 \mathrm{~h}$

$$
\text { (ii),(iii) }
$$

Reagents and conditions: (i) N-Boc-propargylamine (1.1 equiv), Pd(PPh $)_{4}(11 \mathrm{~mol} \%)$, Cul (19 mol \%), NEt 3 (2.0 equiv), THF, $80{ }^{\circ} \mathrm{C}$, $24 \mathrm{~h}$, sealed tube; (ii) TFA (30 equiv), $\mathrm{CH}_{2} \mathrm{Cl}_{2}, \mathrm{rt}, 14 \mathrm{~h}$; (iii) $\mathrm{ArSO}_{2} \mathrm{C}\left(\mathrm{CH}_{3}\right)_{2} \mathrm{COOH}$ (1.1 equiv), $\mathrm{PyBOP}$ (1.1 equiv), $i-\mathrm{Pr}_{2} \mathrm{NEt}(2.3$ equiv), DMF, $0^{\circ} \mathrm{C}$ to ott, $15 \mathrm{~h}$; (iv) Pd/C (17 mol \%), $\mathrm{H}_{2}(1 \mathrm{~atm}), \mathrm{THF}, \mathrm{rt}, 4 \mathrm{~h}$.

Figure S1. Detailed synthetic scheme for the remainder of the ACP1 analogs.

(A-E) Shown are the detailed synthetic routes for the remainder of the ACP1 analogs. 
Table S2. Naming of ACP1 and ADEP analogs.

This table correlates our naming of the compounds with the previously published names.

\begin{tabular}{|c|c|c|}
\hline Compound & Common Name & Reference \\
\hline ACP1-06 & ACP1b & 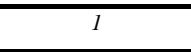 \\
\hline ACP1-44 & ACP1a & 1 \\
\hline ACP1-45 & $\mathrm{ACP} 1$ & 1 \\
\hline ADEP-01 & $\mathrm{n} / \mathrm{a}$ & 2 \\
\hline ADEP-02 & $\mathrm{n} / \mathrm{a}$ & 2 \\
\hline ADEP-03 & A54556 Factor C (ADEP1C) & 3 \\
\hline ADEP-04 & A54556 Factor D (ADEP1D) & 3 \\
\hline ADEP-05 & A54556 Factor A (ADEP1A) & 3 \\
\hline ADEP-06 & $\mathrm{n} / \mathrm{a}$ & 2,4 \\
\hline ADEP-07 & $\mathrm{n} / \mathrm{a}$ & 5 \\
\hline ADEP-08 & A54556 Factor B (ADEP1B) & 3 \\
\hline ADEP-11 & ADEP 4 & 4 \\
\hline ADEP-13 & $\mathrm{n} / \mathrm{a}$ & 5 \\
\hline ADEP-14 & $\mathrm{n} / \mathrm{a}$ & 5 \\
\hline ADEP-15 & $\mathrm{n} / \mathrm{a}$ & 2,6 \\
\hline ADEP-16 & $\mathrm{n} / \mathrm{a}$ & 3 \\
\hline ADEP-17 & $\mathrm{n} / \mathrm{a}$ & 5 \\
\hline ADEP-18 & $\mathrm{n} / \mathrm{a}$ & 4 \\
\hline ADEP-20 & $\mathrm{n} / \mathrm{a}$ & 6 \\
\hline ADEP-21 & $\mathrm{n} / \mathrm{a}$ & 2 \\
\hline ADEP-22 & $\mathrm{n} / \mathrm{a}$ & 3 \\
\hline ADEP-25 & $\mathrm{n} / \mathrm{a}$ & 2 \\
\hline ADEP-26 & A54556 Factor E (ADEP1E) & 3 \\
\hline ADEP-28 & $\mathrm{n} / \mathrm{a}$ & 7 \\
\hline ADEP-29 & $\mathrm{n} / \mathrm{a}$ & 5 \\
\hline ADEP-30 & $\mathrm{n} / \mathrm{a}$ & 5 \\
\hline ADEP-31 & $\mathrm{n} / \mathrm{a}$ & 3 \\
\hline ADEP-32 & $\mathrm{n} / \mathrm{a}$ & 5 \\
\hline ADEP-34 & $\mathrm{n} / \mathrm{a}$ & 3 \\
\hline ADEP-35 & $\mathrm{n} / \mathrm{a}$ & 3 \\
\hline ADEP-36 & A54556 Factor H (ADEP1H) & 3 \\
\hline ADEP-37 & $\mathrm{n} / \mathrm{a}$ & 2 \\
\hline ADEP-38 & $\mathrm{n} / \mathrm{a}$ & 5 \\
\hline ADEP-40 & $\mathrm{n} / \mathrm{a}$ & 3 \\
\hline ADEP-41 & $\mathrm{n} / \mathrm{a}$ & 7 \\
\hline ADEP-42 & $\mathrm{n} / \mathrm{a}$ & 2 \\
\hline ADEP-43 & $\mathrm{n} / \mathrm{a}$ & 2 \\
\hline ADEP-45 & $\mathrm{n} / \mathrm{a}$ & 2 \\
\hline ADEP-46 & $\mathrm{n} / \mathrm{a}$ & 3 \\
\hline
\end{tabular}




\section{REFERENCES}

1. Leung, E., Datti, A., Cossette, M., Goodreid, J., McCaw, S. E., Mah, M., Nakhamchik, A., Ogata, K., El Bakkouri, M., Cheng, Y. Q., Wodak, S. J., Eger, B. T., Pai, E. F., Liu, J., Gray-Owen, S., Batey, R. A., and Houry, W. A. (2011) Activators of cylindrical proteases as antimicrobials: identification and development of small molecule activators of ClpP protease, Chemistry \& biology 18, 1167-1178.

2. Goodreid, J. D., dos Santos Eda, S., and Batey, R. A. (2015) A Lanthanide(III) Triflate Mediated Macrolactonization/Solid-Phase Synthesis Approach for Depsipeptide Synthesis, Organic letters 17, 2182-2185.

3. Goodreid, J. D., Wong, K., Leung, E., McCaw, S. E., Gray-Owen, S. D., Lough, A., Houry, W. A., and Batey, R. A. (2014) Total synthesis and antibacterial testing of the A54556 cyclic acyldepsipeptides isolated from Streptomyces hawaiiensis, Journal of natural products 77, 2170-2181.

4. Hinzen, B., Raddatz, S., Paulsen, H., Lampe, T., Schumacher, A., Habich, D., Hellwig, V., Benet-Buchholz, J., Endermann, R., Labischinski, H., and Brotz-Oesterhelt, H. (2006) Medicinal chemistry optimization of acyldepsipeptides of the enopeptin class antibiotics, ChemMedChem 1, 689-693.

5. Goodreid, J. D., Janetzko, J., Santa Maria, J. P., Jr., Wong, K. S., Leung, E., Eger, B. T., Bryson, S., Pai, E. F., Gray-Owen, S. D., Walker, S., Houry, W. A., and Batey, R. A. (2016) Development and Characterization of Potent Cyclic Acyldepsipeptide Analogues with Increased Antimicrobial Activity, Journal of medicinal chemistry 59, 624-646.

6. Hinzen, B., Brötz-Oesterhelt, H., Endermann, R., Henninger, K., Paulsen, H., Raddatz, S., Lampe, T., Hellwig, V., and Schumacher, A. B. A. (2005) Antibacterial macrocycles, (Patent, U., Ed.), USA.

7. Carney, D. W., Schmitz, K. R., Truong, J. V., Sauer, R. T., and Sello, J. K. (2014) Restriction of the conformational dynamics of the cyclic acyldepsipeptide antibiotics improves their antibacterial activity, Journal of the American Chemical Society 136, 1922-1929. 